\title{
The oxidation of 3-alkylhomophthalimide derivatives by dioxygen in alkaline media and Bischler-Napieralski reactions of cleavage- cyclisation products
}

\author{
Harry Heaney* and Mutasem O. Taha \\ Department of Chemistry, Loughborough University, Loughborough, \\ Leicestershire LE11 3TU, UK \\ E-mail: h.heaney@lboro.ac.uk
}

\section{Dedicated to Professor Gurnos Jones on the occasion of his 70th birthday - a good friend since 1955}

(received 03 Mar 00; accepted 20 Aug 00; published on the web 28 Aug 00)

\begin{abstract}
The oxidation of 4-alkylhomophthalimide derivatives by dioxygen in the presence of ethanolic sodium hydroxide leads to the rapid formation of $\alpha$-hydroxy- $\alpha$-alkylhomophthalimide derivatives and hence, as a result of ring cleavage and lactone formation, to $\alpha$-amido- $\alpha$-alkylphthalides that can be converted into analogues of phthalideisoquinoline alkaloids.
\end{abstract}

Keywords: Alkylhomophthalimide oxidation, dioxygen, Bischler-Napieralski reactions, phthalideisoquinoline alkaloids

\section{Introduction}

The chemistry of dioxygen is of considerable current interest in view of its impact on our understanding of a range of chemical and biological reactions. ${ }^{1}$ The cleavage of ketones by oxygen as a result of the formation of $\alpha$-hydroperoxyketones has been reported many times, particularly when the ketone forms a stable enol. ${ }^{2}$ Even simple ketones have been shown to react with oxygen in a strongly basic medium. ${ }^{3}$ The autoxidation of ketones can take place without the cleavage of the carbon chain as exemplified by the $\alpha$-hydroxylation of ()-isorotenone, ${ }^{4}$ a number of steroidal 20 -ketones, ${ }^{5}$ and sesquiterpenoids ${ }^{6}$ and triterpenoids, ${ }^{7}$ as well as the g-hydroxylation of $\alpha, \beta$-unsaturated ketones such as cyperone. ${ }^{8}$ The direct oxygenation of enolates, generated by anionic oxy-Cope rearrangement reactions, has been reported more recently in connection with the synthesis of polycyclic $\alpha$-hydroxy ketones, ${ }^{9}$ and hydroxylation $\alpha$ - to an aryl ketone has also been reported as part of the pinene pathway to taxanes. ${ }^{10}$ Other carbonyl compounds such as esters $^{11}$ and dialkylmaleic anhydrides ${ }^{12}$ have also been shown to give autoxidation products that 
involve reactions of carbanions. The ease of formation of 3-hydroxy-1,3-dimethyl-5methoxyoxindole during the asymmetric alkylation of 1,3-dimethyl-5-methoxy-oxindole may well be related to the aromatic character of the enolate of the amide function. ${ }^{13}$ The autoxidation of esters and amides to the related $\alpha$-hydroxy-derivatives has been studied using titanium enolates $^{14}$ and a recent report is concerned with the oxidation of anions derived from a series of b-imidoesters using dibenzoyl peroxide. ${ }^{15}$ It has been pointed out that the ease of oxidation of ionizable organic compounds depends not only on the degree of conversion to an anion but also on the relative stabilities of the carbanions and the related radicals. ${ }^{16}$ For example, in a b-dicarbonyl system the enolate ion is more stabilized than the related radical and hence the latter is more difficult to form. It is therefore entirely in accord with expectation that the b-imidoesters could not be oxidised cleanly by dioxygen. ${ }^{15}$ As far as we are aware there was only one report ${ }^{17}$ of reactions of homophthalimide derivatives with dioxygen prior to our preliminary publication, ${ }^{18}$ although the dye sensitised photooxygenation of homophthalimides has been reported more recently. ${ }^{19}$ The earlier study related to reactions of products formed in ring opening reactions of 4-spirocyclopropane derivatives with amines. ${ }^{17}$ The oxidation reactions were reported to occur spontaneously in the solid state in reactions that clearly depend on the presence of an intramolecular base as shown in Scheme 1. We now record the full details of our observations concerning the autoxidation of some 4-alkylisoquinoline-1,3(2H,4H)-diones and the conversion of two lactone-amides into a phthalide-dihydroisoquinoline and a phthalide-dihydrob-carboline.

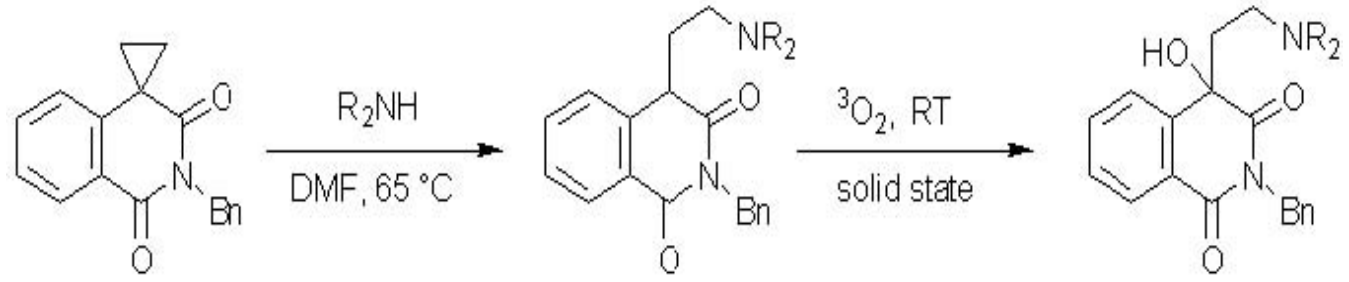

\section{Scheme 1}

The formation of enolate ions from $\mathrm{N}$-substituted derivatives of homophthalimide is particularly easy and has been used by us previously in order to introduce two alkyl residues at the benzylic carbon. ${ }^{20}$ In the case of the reaction of $N$-benzylhomophthalimide with methyl iodide in aqueous ethanolic sodium hydroxide the reaction proceeds rapidly with the formation of the dimethyl derivative. The greater steric requirement of the isopropyl group suggested that the formation of the monoalkylated derivative (1) would be straightforward and this has been confirmed by experiment. A reaction of $N$-benzylhomophthalimide with isopropyl iodide in aqueous ethanolic sodium hydroxide gave the expected product (1) in $42 \%$ yield. On the other hand when we carried out analogous reactions using either ethyl iodode or benzyl bromide we obtained mixtures of the mono- and di-alkylated products which were separated by flash chromatography on silica gel.

When a solution of (1) in ethanolic sodium hydroxide was saturated with dioxygen and then heated under reflux the fluorescence was discharged after about three hours and gave a 
quantitative yield of a product that had incorporated an additional oxygen atom and where the two carbonyl stretching frequencies in the infrared spectrum of the original homophthalimide ( $\mathrm{n}_{\max } 1670$ and $1714 \mathrm{~cm}^{-1}$ ) had been replaced by two new absorptions ( $\mathrm{n}_{\max } 1676$ and $1773 \mathrm{~cm}^{-1}$ ). An accurate mass measurement on the mass spectrometric molecular ion established the molecular formula and the ${ }^{1} \mathrm{H}$ and ${ }^{13} \mathrm{C}$ nuclear magnetic resonance spectra confirmed the structure of the lactone (2) in which a ring contraction from the six-membered to a fivemembered ring had occurred (Scheme 2). It is important to note that the reactions do not proceed in the absence of base, confirming our interpretation of the importance of the b-aminoethylresidues in the reactions exemplified in Scheme $1 .{ }^{17}$ Ring contraction reactions from a sixmembered to a five-membered ring have been reported previously, and are exemplified for example by the conversion of $\mathrm{N}$-substituted isoquinolinetriones into ethyl 3-hydroxyisoindolone3 -carboxylates by heating in ethanol in the presence of triethylamine. ${ }^{21}$

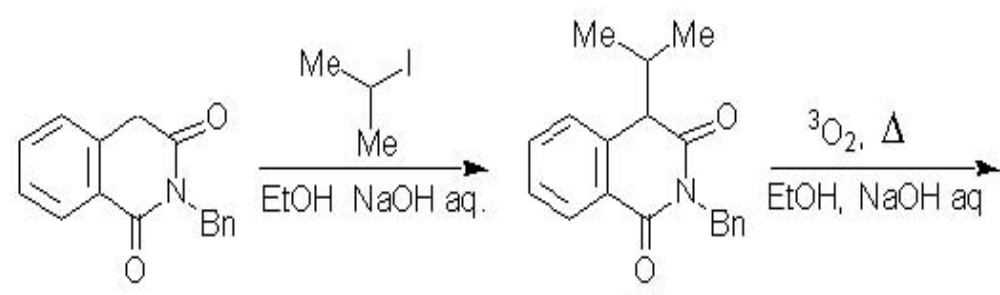

(1)

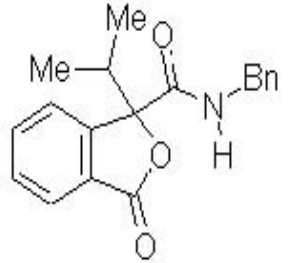

(2)

\section{Scheme 2}

The reaction conditions used suggested the intermediacy of a tertiary hydroperoxide but, as with the $\alpha$-ketohydroperoxide derived from cyclohexanone which is apparently very unstable, ${ }^{22}$ we were unable to detect a hydroperoxide by the starch-iodide test. The latter result suggested that the reaction could be catalytic in base and a reaction in the presence of $15 \mathrm{~mol} \%$ of sodium hydroxide also gave the lactone (2) in high yield. By carrying out the oxidation reaction using a catalytic amount of sodium hydroxide in the presence of an excess of triethyl phosphite ${ }^{23}$ and a continuous supply of dioxygen, we were able to stop the isomerisation of the proposed hydroxyhomophthalimide (3). In the latter reaction (Scheme 3) the compound (3) was isolated in $97 \%$ yield together with a small amount of recovered starting material (1).<smiles>[Y6]C(C)C1C(=O)N(Cc2ccccc2)C(=O)c2ccccc21</smiles>

(1)

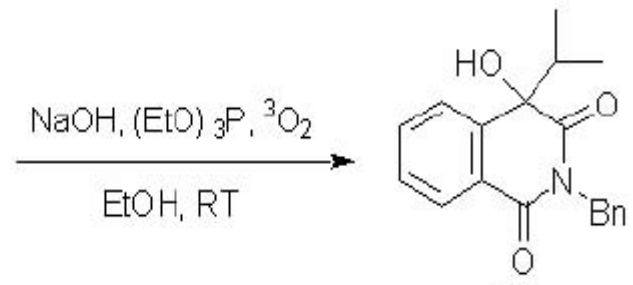

(3)

\section{Scheme 3}


It is clear that a number of alternative routes are available to the initial radical (B), for example the involvement of the hydroxyl radical in removing an electron from the anion would also regenerate hydroxide ion. Similarly the radical (B) would be generated by interaction of the starting material (A) with the oxy-radical (C). A mechanistic sequence that accounts for the observed results is shown in Scheme 4.<smiles>[R]C1C(=O)N([R])C(=O)c2ccccc2C1[R]</smiles>

(A)<smiles>[R]NC(=O)C1([R])OC(=O)c2ccccc21</smiles><smiles>[R]N1C(=O)c2ccccc2C([R])(O)C1=O</smiles>

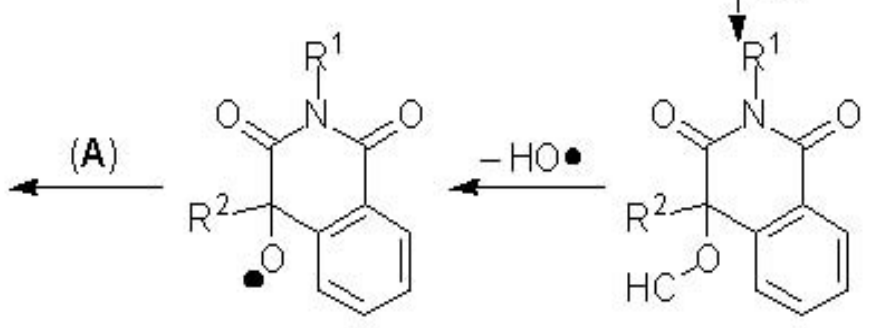<smiles>[R2]C1C(=O)N([R7])C(=O)c2ccccc21</smiles>

(B)<smiles>[R]N1C(=C)C([R])(OC)c2ccccc2C1=O</smiles>

(A)

(C)

\section{Scheme 4}

We found that the ethyl analogue (4) of the compound (1) is converted more slowly than the compound (1) and gave, after $9 \mathrm{~h}$, a similar lactone (6) in $25 \%$ yield together with a second product that was formed in $36 \%$ yield in a reaction using a catalytic quantity of sodium hydroxide. The products were separated by flash chromatography and the second product was shown to be the expected intermediate hydroxyhomophthalimide (5). The hydroxyhomophthalimide (5) was converted into the phthalide derivative (6) in a quantitative yield when it was heated under reflux in ethanolic sodium hydroxide solution. The benzyl derivative (7) was shown to react even more slowly than the ethyl derivative (4) and gave the hydroxy compound (8) in 33\% yield together with the lactone (9) in 11\% yield and unchanged starting material $(56 \%)$ when the reaction was allowed to proceed for $17 \mathrm{~h}$ using a catalytic quantity of sodium hydroxide. A substituent is clearly required on the benzylic carbon in a homophthalimide for the autoxidation to proceed; we found that $N$-benzylhomophthalimide does not undergo the autoxidation reaction under the conditions reported herein. 
<smiles>[R]C1C(=O)N(Cc2ccccc2)C(=O)c2ccccc21</smiles><smiles>[R]C1(O)C(=O)N(Cc2ccccc2)C(=O)c2ccccc21</smiles><smiles>[R]C1(C(=O)NCc2ccccc2)OC(=O)c2ccccc21</smiles>
(4, R= Et)
$(5, R=E t)$
(6, $R=E t)$
$(7, \mathrm{R}=\mathrm{Bn})$
$(8, R=B n)$
$(9, \mathrm{R}=\mathrm{Bn})$

The preparation of lactonic amide precursors for Bischler-Napieralski cyclisation reactions that can lead to phthalide-isoquinoline alkaloids ${ }^{24}$ is frequently troublesome. ${ }^{25}$ It is clear that our new procedure provides a straightforward route to amidophthalides which, if the benzyl group in, for example ( $\mathbf{2}$ or $\mathbf{6}$ ), was replaced by a b-arylethyl residue, could function as key intermediates in Bischler-Napieralski cyclisation reactions en route to analogues of phthalideisoquinoline alkaloids. The natural products have been shown to be potent central nervous system active compounds. ${ }^{26}$ A number of other phthalides have also been shown to have other interesting biological properties. ${ }^{27}$ Two representative homophthalimide derivatives were prepared in order to test their suitability for Bischler-Napieralski cyclisation reactions. Thus, 3,4-dimethoxy-bphenylethylamine and tryptamine were converted into the related $N$-substituted homophthalimide derivatives (10) and (11) respectively. Alkylation of the compound (10) with ethyl iodide gave a mixture of the mono- (12) and di-alkylated (13) products in $29 \%$ and $9 \%$ yields respectively while a similar reaction of the compound (11) using isopropyl iodide gave the expected 2-[2(1H-3-indolyl)ethyl]-4-isopropyl-1,2,3,4-tetrahydro-1,3-isoquinolinedione (14) in 44\% yield. Oxidation of 2-(3,4-dimethoxyphenethyl)-4-ethyl-1,2,3,4-tetrahydro-1,3-isoquinolinedione (12) using the standard procedure gave a mixture of products that were separated by flash chromatography and shown to be 2-(3,4-dimethoxyphenethyl)-4-ethyl-4-hydroxy-1,2,3,4tetrahydro-1,3-isoquinolinedione(15) and the phthalide derivative, $N-1-(3,4-$ dimethoxyphenethyl)-1-ethyl-3-oxo-1,3-dihydro-1-isobenzofurancarboxamide,(16) which were isolated in $10 \%$ and $51 \%$ yields respectively. As we anticipated, the oxidation of the indole derivative (14) proceeded more rapidly and gave an excellent yield (91\%) of $\mathrm{N}-1-[2-(1 \mathrm{H}-3-$ indolyl)ethyl]-1-isopropyl-3-oxo-1,3-dihydro-1-isobenzofurancarboxamide (17). Finally, we carried out BischlerNapieralski cyclisation reactions using the phthalide derivatives (16) and (17) by heating them with an excess of phosphoryl chloride which gave the 3,4-dihydroisoquinoline derivatives (18) and (19) in 58\% and 22\% yields respectively. 

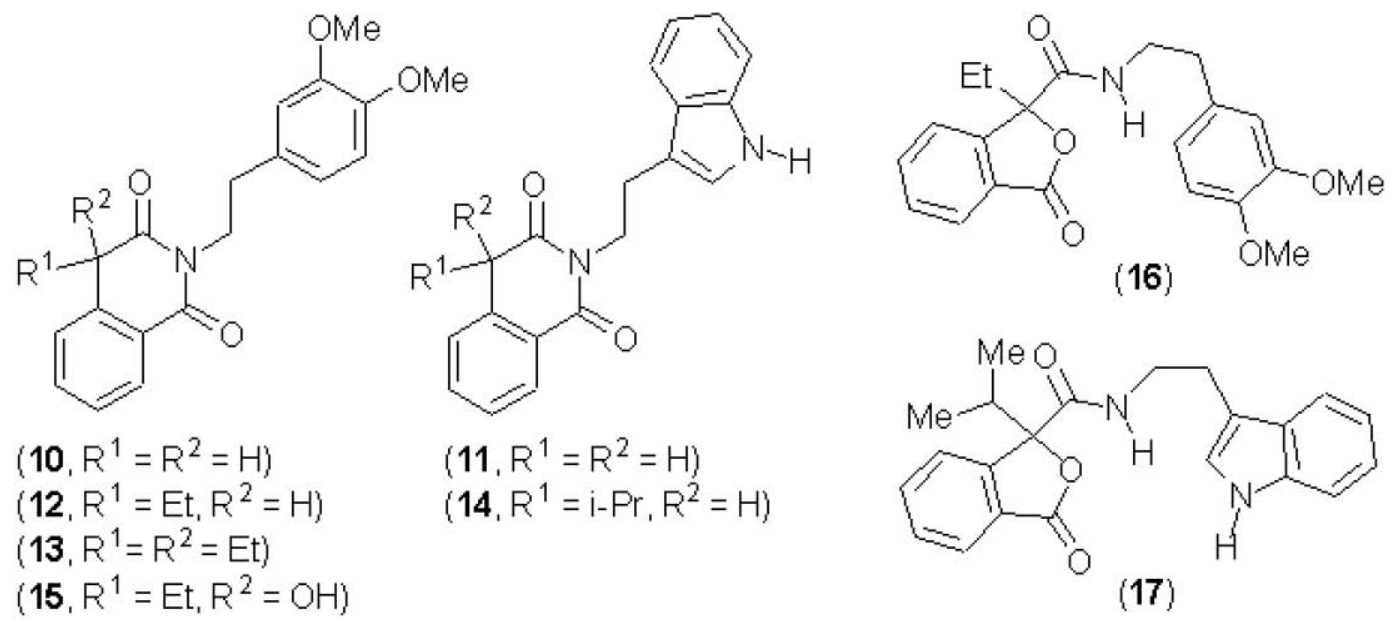

(16)<smiles>CC(C)C1(C(=O)NCCc2c[nH]c3ccccc23)OC(=O)c2ccccc21</smiles>

(17)<smiles>CCC1(C(=O)NCCc2ccc(OC)c(OC)c2)OC(=O)c2ccccc21</smiles>

(16)<smiles>CC(C)C1(C(=O)NCCc2c[nH]c3ccccc23)OC(=O)c2ccccc21</smiles>

(17)<smiles>CCC1(C2=NCCc3cc(OC)c(OC)cc32)OC(=O)c2ccccc21</smiles>

(18)

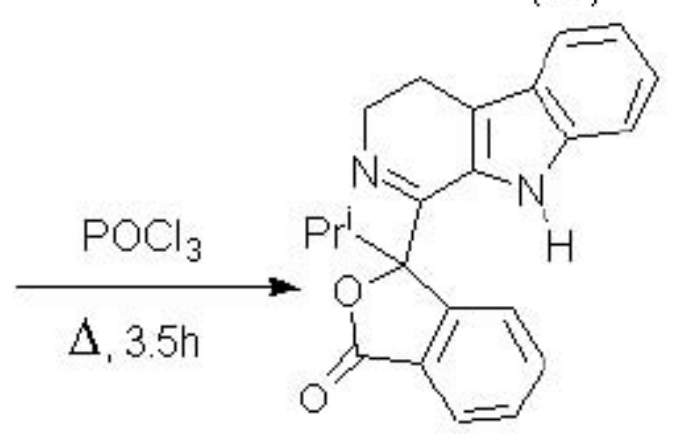

(19)

In conclusion, we have developed a mild protocol that allows the direct oxygenation of tertiary benzylic imide centres that not only produces compounds of potential biological interest, but also produces phthalides by ring cleavage and recyclisation that can be used as precursors to analogues of phthalideisoquinoline alkaloids.

\section{Experimental Section}

General Procedures. Commercially available solvents and reagents were used throughout without further purification, except for those detailed below which were purified as described. Light petroleum refers to the fraction boiling between $40^{\circ} \mathrm{C}$ and $60^{\circ} \mathrm{C}$. Light petroleum and ethyl 
acetate were distilled from anhydrous calcium chloride through a $36 \mathrm{~cm}$ Vigreux column before use. Dichloromethane was distilled from phosphorus pentoxide.

Chromatographic procedures - Analytical thin layer chromatography was carried out using glass-backed plates coated in Merck Kieselgel $60 \mathrm{PF}_{254}$. Plates were visualised by UV light (at $254 \mathrm{and} /$ or $360 \mathrm{~nm}$ ) or by exposure to an appropriate staining agent. Flash chromatography was carried out using Merck silica gel 60. Pressure was applied at the head of the column with hand bellows. Samples were applied as a concentrated solution in an appropriate solvent.

Spectroscopic techniques - Infared spectra were recorded in the range $4000-600 \mathrm{~cm}^{-1}$ using a Nicolet FT-205 spectrometer with internal calibration. Spectra were recorded as thin films or as Nujol mulls. ${ }^{1} \mathrm{H}$ and ${ }^{13} \mathrm{C}$ NMR spectra were recorded using Bruker AC-250 and Bruker DPX-400 instruments. ${ }^{1} \mathrm{H}$ NMR spectra are referenced against residual undeuterated solvent, in the case of deuteriochlorofom this is $7.260 \mathrm{ppm}$. Signals are described as singlets (s), doublets (d), double doublet (dd), quartets (q), multiplet (m) etc. High and low resolution mass spectra were recorded on a Kratos MS80 instrument.

Other data and instrumentation - Melting points were measured on a Electrothermal digital melting point apparatus or using a Kofler hot stage apparatus and are uncorrected. Bulb to bulb distillations were performed on a Büchi GKR-51 Kugelrohr; boiling points refer to air bath temperatures and are uncorrected.

All of the following experimental reactions were carried out under an atmosphere of nitrogen except where it was obviously unnecessary. The modulus of coupling constants $(J)$ are quoted in Hertz.

\section{General method for 1,2,3,4-tetrahydro-1,3-isoquinolinediones formation}

The amine ( 1 equivalent) was added to homophthalic anhydride and stirred at $70{ }^{\circ} \mathrm{C}$. The reaction mixture was then heated to the stated temperature for a given length of time. After allowing the mixture to cool to room temperature, the product was purified either by recrystallisation or by flash chromatography on silica gel. The following 1,3-isoquinolinediones were prepared:

2-Benzyl-1,2,3,4-tetrahydro-1,3-isoquinolinedione. Benzylamine (1.0g, $9.3 \mathrm{mmol})$ and homophthalic anhydride $(1.5 \mathrm{~g}, 9.3 \mathrm{mmol})$ were heated at $185^{\circ} \mathrm{C}$ for 30 minutes and gave the title compound (2.3g, 100\%), mp 110-112 ${ }^{\circ} \mathrm{C}$ (glass) (lit. ${ }^{28} 124-126{ }^{\circ} \mathrm{C}$ ) : $\mathrm{v}_{\max } 3035,1716$ and $1670 \mathrm{~cm}^{-1} ;{ }^{1} \mathrm{H}$ NMR $\left(250 \mathrm{M} \mathrm{Hz}, \mathrm{CDCl}_{3}\right) \delta 3.98(\mathrm{~s}, 2 \mathrm{H}), 5.14(\mathrm{~s}, 2 \mathrm{H}), 7.17-7.52(\mathrm{~m}, 8 \mathrm{H})$ and 8.15-8.18 (m, 1H) ppm; ${ }^{13} \mathrm{C}$ NMR $\left(100.5 \mathrm{M} \mathrm{Hz}, \mathrm{CDCl}_{3}\right) 37.04\left(\mathrm{CH}_{2}\right), 43.84\left(\mathrm{CH}_{2}\right), 125.95(\mathrm{C})$, $127.71(\mathrm{CH}), 128.26(\mathrm{CH}), 128.38(\mathrm{CH}), 128.99(2 \times \mathrm{CH}), 129.22(2 \times \mathrm{CH}), 129.79(\mathrm{CH})$, $134.22(\mathrm{CH}), 134.75(\mathrm{C}), 137.71(\mathrm{C}), 165.25(\mathrm{C}=\mathrm{O})$ and $170.44(\mathrm{C}=\mathrm{O}) \mathrm{ppm}$; Found: $\mathrm{M} 251.0950$ (88.5\%) Calc. for $\mathrm{C}_{16} \mathrm{H}_{13} \mathrm{NO}_{2} 251.0946$.

2-(3,4-Dimethoxyphenethyl)-1,2,3,4-tetrahydro-1,3-isoquinolinedione. 2-(3,4-Dimethoxyphenyl)ethylamine $(1.1 \mathrm{~g}, 6.2 \mathrm{mmol})$ and homophthalic anhydride $(1.0 \mathrm{~g}, 6.2 \mathrm{mmol})$ were heated at $185{ }^{\circ} \mathrm{C}$ for 30 minutes and gave the title compound (10) after flash chromatography eluting with ethyl acetate : 
light petroleum [1:1] (1.8 g, 90\%), mp $145-146.4{ }^{\circ} \mathrm{C}$ (lit. $\left.{ }^{29} 147-148{ }^{\circ} \mathrm{C}\right)$ from ethyl acetate : light petroleum: $\mathrm{v}_{\max } 3055,1716$ and $1668 \mathrm{~cm}^{-1} ;{ }^{1} \mathrm{H} \mathrm{NMR}\left(250 \mathrm{M} \mathrm{Hz}, \mathrm{CDCl}_{3}\right) \delta 2.81-2.87(\mathrm{~m}, 2 \mathrm{H})$, $3.83(\mathrm{~s}, 3 \mathrm{H}, \mathrm{OMe}), 3.84(\mathrm{~s}, 3 \mathrm{H}, \mathrm{OMe}), 3.99(\mathrm{~s}, 2 \mathrm{H}), 4.13-4.20(\mathrm{~m}, 2 \mathrm{H}), 6.76-6.81(\mathrm{~m}, 3 \mathrm{H}), 7.22-$ $7.56(\mathrm{~m}, 3 \mathrm{H})$ and 8.17-8.20 (m, 1H) ppm; ${ }^{13} \mathrm{C} \mathrm{NMR}\left(62.8 \mathrm{M} \mathrm{Hz}, \mathrm{CDCl}_{3}\right) \delta 33.61\left(\mathrm{CH}_{2}\right), 36.29$ $\left(\mathrm{CH}_{2}\right), 41.53\left(\mathrm{CH}_{2}\right), 55.74\left(\mathrm{CH}_{3}, \mathrm{OMe}\right), 55.78\left(\mathrm{CH}_{3}, \mathrm{OMe}\right), 111.11(\mathrm{CH}), 111.99(\mathrm{CH}), 120.85$ $(\mathrm{CH}), 125.26(\mathrm{C}), 127.06(\mathrm{CH}), 127.66(\mathrm{CH}), 128.96(\mathrm{CH}), 131.03(\mathrm{C}), 133.54(\mathrm{CH}), 134.01$ (C), $147.50(\mathrm{C}), 148.72(\mathrm{C}), 164.64(\mathrm{C}=\mathrm{O})$ and $169.73(\mathrm{C}=\mathrm{O}) \mathrm{ppm}$; Found: $\mathrm{C}, 69.51, \mathrm{H}, 6.05, \mathrm{~N}$, 4.41\% Calc. for $\mathrm{C}_{19} \mathrm{H}_{19} \mathrm{NO}_{4} \mathrm{C}, 70.15, \mathrm{H}, 5.84$, N, 4.31\%; Found: M 325.1318 (8.0 \%) Calc. for $\mathrm{C}_{19} \mathrm{H}_{19} \mathrm{NO}_{4} 325.1314$.

2-[2-(1H-3-Indolyl)ethyl]-1,2,3,4-tetrahydro-1,3-isoquinolinedione. Tryptamine $\quad(1.0 \mathrm{~g}$, $6.2 \mathrm{mmol})$ and homophthalic anhydride $(1.0 \mathrm{~g}, 6.2 \mathrm{mmol})$ were heated at $185^{\circ} \mathrm{C}$ for 30 minutes and gave the title compound (11) $(1.6 \mathrm{~g}, 85 \%)$, mp 192-194.5 ${ }^{\circ} \mathrm{C}$ (lit. $\left.{ }^{30} 195-196{ }^{\circ} \mathrm{C}\right)$ from DCM: $\mathrm{v}_{\max } 3370$ (broad), 3154, 1708, and $1655 \mathrm{~cm}^{-1}$; ${ }^{1} \mathrm{H}$ NMR (400 M Hz, $\left.\mathrm{CDCl}_{3}\right) \delta$ 3.10-3.14 (m, 2H), 4.04 (s, 2H), 4.31-4.35 (m, 2H), 7.13-7.88 (m, 8H), 8.00 (br.s, 1H, N-H) and 8.26-8.28 (m, 1H) ppm; ${ }^{13} \mathrm{C}$ NMR $\left(100.5 \mathrm{M} \mathrm{Hz}, \mathrm{CDCl}_{3}\right) \delta 24.30\left(\mathrm{CH}_{2}\right), 36.85\left(\mathrm{CH}_{2}\right), 41.30\left(\mathrm{CH}_{2}\right), 111.39$ $(\mathrm{CH}), 113.43(\mathrm{C}), 119.58(\mathrm{CH}), 119.89(\mathrm{CH}), 122.44(\mathrm{CH}), 122.45(\mathrm{CH}), 125.92(\mathrm{C}), 127.48$ $(\mathrm{CH}), 128.00(\mathrm{C}), 128.10(\mathrm{CH}), 129.52(\mathrm{CH}), 133.94(\mathrm{CH}), 134.53(\mathrm{C}), 136.64(\mathrm{C}), 165.26$ $(\mathrm{C}=\mathrm{O})$ and $170.33(\mathrm{C}=\mathrm{O})$ ppm; Found: $\mathrm{M} 304.1209(8.8 \%)$ Calc. for $\mathrm{C}_{19} \mathrm{H}_{16} \mathrm{~N}_{2} \mathrm{O}_{2}$ 304.1212.

\section{Reactions of 1,2,3,4-tetrahydro-1,3-isoquinolinediones with alkyl- or arylalkyl halides (1} equivalent) and aqueous alcoholic sodium hydroxide (1 equivalent). General method

A magnetically stirred solution of the particular 1,3-isoquinolinedione, sodium hydroxide (1 equivalent) and the appropriate alkylating agent ( 1 equivalent, unless otherwise stated) in aqueous ethanolic solution [1:1] ( $c a .150 \mathrm{~mL}$ ) was heated under reflux for a given length of time. Subsequently, the reaction mixture was allowed to cool to room temperature then DCM $(150 \mathrm{~mL})$ and water $(150 \mathrm{~mL})$ were added to the reaction mixture followed by vigorous shaking. The organic layer was then collected and the aqueous layer was further extracted with DCM $(100 \mathrm{~mL})$. The combined organic extracts were dried over magnesium sulfate, concentrated in vacuo and the residue was purified by flash chromatography on silica gel and/or recrystallisation. The following alkylation reactions were conducted:

Reaction of 2-benzyl-1,2,3,4-tetrahydro-1,3-isoquinolinedione with ethyl iodide and $\mathrm{NaOH}$ A stirred solution of 2-benzyl-1,2,3,4-tetrahydro-1,3-isoquinolinedione (1.5 g, $6.0 \mathrm{mmol})$, sodium hydroxide $(0.24 \mathrm{~g}, 6.0 \mathrm{mmol})$ and ethyl iodide $(0.94 \mathrm{~g}, 6.0 \mathrm{mmol})$ in aqueous ethanol [1:1] (ca. $140 \mathrm{ml}$ ) was heated under reflux for 6 hours and gave two products after flash chromatography eluting with ethyl acetate : light petroleum [1:5]. The first product 2-benzyl-4,4diethyl-1,2,3,4-tetrahydro-1,3-isoquinolinedione (0.22 g, 12\%), mp 44-46 ${ }^{\circ} \mathrm{C}$ from ethyl acetate : light petroleum: $\mathrm{v}_{\max } 2967,1712,1669$ and $1606 \mathrm{~cm}^{-1} ;{ }^{1} \mathrm{H} \mathrm{NMR}\left(250 \mathrm{M} \mathrm{Hz}, \mathrm{CDCl}_{3}\right) \delta 0.42(\mathrm{t}$, $6 \mathrm{H}, 2 \times \mathrm{CH}_{3}, J$ 7.4), 1.81-1.89 (m, 2H), 2.26-2.34 (m, 2H), $5.20(\mathrm{~s}, 2 \mathrm{H}), 7.19-7.63$ (m, 8H) and 8.24-8.27 (m, 1H) ppm; ${ }^{13} \mathrm{C}$ NMR $\left(68.2 \mathrm{M} \mathrm{Hz} \mathrm{CDCl}_{3}\right) \delta 9.19\left(2 \times \mathrm{CH}_{3}\right), 36.19\left(2 \times \mathrm{CH}_{2}\right), 43.58$ $\left(\mathrm{CH}_{2}\right), 53.92(\mathrm{C}), 125.04(\mathrm{CH}), 126.81(\mathrm{C}), 127.20(\mathrm{CH}), 127.39(\mathrm{CH}), 128.36(2 \times \mathrm{CH}), 128.81$ 
$(\mathrm{CH}), 128.87(2 \times \mathrm{CH}), 134.18(\mathrm{CH}), 137.35(\mathrm{C}), 141.97(\mathrm{C}), 164.37(\mathrm{C}=\mathrm{O})$ and $175.97(\mathrm{C}=\mathrm{O})$ ppm; Found: M307.1581 (56.6\%) $\mathrm{C}_{20} \mathrm{H}_{21} \mathrm{NO}_{2}$ requires 307.1572. The second product 2-benzyl4-ethyl-1,2,3,4-tetrahydro-1,3-isoquinoline-dione(4) (0.44 g, 26\%), yellow oil : $\mathrm{v}_{\max } 2969,1715$, 1670 and $1603 \mathrm{~cm}^{-1}$; ${ }^{1} \mathrm{H}$ NMR $\left(400 \mathrm{M} \mathrm{Hz}, \mathrm{CDCl}_{3}\right) \delta 0.70(\mathrm{t}, 3 \mathrm{H}, J$ 7.4), 1.98-2.05 (m, 1H), 2.22$2.30(\mathrm{~m}, 1 \mathrm{H}), 3.95(\mathrm{t}, 1 \mathrm{H}, J 5.5), 5.20\left(\mathrm{q}, 2 \mathrm{H}, \mathrm{AB}, J_{\mathrm{AB}} 13.8\right), 7.24-7.62(\mathrm{~m}, 8 \mathrm{H})$ and 8.24-8.26 (m, 1H) ppm; ${ }^{13} \mathrm{C}$ NMR $\left(100.5 \mathrm{M} \mathrm{Hz}, \mathrm{CDCl}_{3}\right) \delta 9.77\left(\mathrm{CH}_{3}\right), 30.81\left(\mathrm{CH}_{2}\right), 43.75\left(\mathrm{CH}_{2}\right), 47.63(\mathrm{CH})$, $126.16(\mathrm{C}), 127.18(\mathrm{CH}), 127.84(\mathrm{CH}), 127.93(\mathrm{CH}), 128.77(2 \times \mathrm{CH}), 129.31(2 \times \mathrm{CH}), 129.39$ $(\mathrm{CH}), 134.05(\mathrm{CH}), 137.62(\mathrm{C}), 139.30(\mathrm{C}), 165.13(\mathrm{C}=\mathrm{O})$ and $173.97(\mathrm{C}=\mathrm{O})$ ppm; Found: M279.1257 (100.0\%) $\mathrm{C}_{18} \mathrm{H}_{17} \mathrm{NO}_{2}$ requires 279.1259 .

Reaction of 2-(3,4-dimethoxyphenethyl)-1,2,3,4-tetrahydro-1,3-isoquinoline dione with ethyl iodide and NaOH. A stirred solution of 2-(3,4-dimethoxyphenethyl)-1,2,3,4-tetrahydro1,3-isoquinolinedione (10) (3.3 g, $10.0 \mathrm{mmol})$, sodium hydroxide $(0.40 \mathrm{~g}, 10.0 \mathrm{mmol})$ and ethyl iodide $(1.6 \mathrm{~g}, 10.0 \mathrm{mmol})$ in aqueous ethanol [1:1] (ca. $120 \mathrm{~mL})$ was heated under reflux for 3.5 hours and gave two products after flash chromatography eluting with ethyl acetate : light petroleum [1:5]. The first product 2-(3,4-dimethoxyphenethyl)-4,4-diethyl-1,2,3,4-tetrahydro-1,3isoquinolinedione (13) (0.35 g, 9\%), mp 87-89 ${ }^{\circ} \mathrm{C}$ from ethyl acetate : light petroleum: $\mathrm{v}_{\max }$ 2967, 1711, 1667 and $1607 \mathrm{~cm}^{-1} ;{ }^{1} \mathrm{H} \mathrm{NMR}\left(250 \mathrm{M} \mathrm{Hz}, \mathrm{CDCl}_{3}\right) \delta 0.45$ (t, 6H, $\left.2 \times \mathrm{CH}_{3}, J 7.4\right)$, 1.80-1.89 (m, 2H), 2.24-2.32 (m, 2H), 2.81-2.88 (m, 2H), 3.81 (s, 3H, OMe), 3.84 (s, 3H, OMe), 4.17-4.29 (m, 2H), 6.78-6.83 (m, 3H), 7.23-7.65 (m, 3H) and 8.22-8.25 (m, 1H) ppm; ${ }^{13} \mathrm{C} \mathrm{NMR}$ $\left(62.8 \mathrm{M} \mathrm{Hz}, \mathrm{CDCl}_{3}\right) \delta 9.19\left(2 \times \mathrm{CH}_{3}\right), 33.80\left(\mathrm{CH}_{2}\right), 36.05\left(2 \times \mathrm{CH}_{2}\right), 41.86\left(\mathrm{CH}_{2}\right), 53.81(\mathrm{C})$, $55.83\left(\mathrm{CH}_{3}, \mathrm{OMe}\right), 55.91\left(\mathrm{CH}_{3}, \mathrm{OMe}\right), 111.34(\mathrm{CH}), 112.16(\mathrm{CH}), 120.96(\mathrm{CH}), 125.04(\mathrm{CH})$, $126.76(\mathrm{C}), 127.20(\mathrm{CH}), 128.53(\mathrm{CH}), 131.22(\mathrm{C}), 134.10(\mathrm{CH}), 141.91(\mathrm{C}), 147.65(\mathrm{C}), 148.91$ $(\mathrm{C}), 164.21(\mathrm{C}=\mathrm{O})$ and $175.80(\mathrm{C}=\mathrm{O})$ ppm; Found: $\mathrm{M} 381.1939(59.4 \%) \mathrm{C}_{23} \mathrm{H}_{27} \mathrm{NO}_{4}$ requires 381.1940. The second product 2-(3,4-dimethoxyphenethyl)-4-ethyl-1,2,3,4-tetrahydro-1,3isoquinolinedione (12) (1.0 g, 29\%), yellow oil : $\mathrm{v}_{\max } 2966,1713,1668$ and $1608 \mathrm{~cm}^{-1}$; ${ }^{1} \mathrm{H}$ NMR $\left(250 \mathrm{M} \mathrm{Hz}, \mathrm{CDCl}_{3}\right) \delta 0.69(\mathrm{t}, 3 \mathrm{H}, J 7.4), 1.92-1.95(\mathrm{~m}, 1 \mathrm{H}), 2.15-2.18(\mathrm{~m}, 1 \mathrm{H}), 2.85-2.92(\mathrm{~m}$, $2 \mathrm{H}), 3.79-3.88(\mathrm{~m}, 1 \mathrm{H}), 3.82(\mathrm{~s}, 3 \mathrm{H}, \mathrm{OMe}), 3.84(\mathrm{~s}, 3 \mathrm{H}, \mathrm{OMe}), 4.10-4.24(\mathrm{~m}, 2 \mathrm{H})$, 6.75-6.84 (m, $3 \mathrm{H}), 7.25-7.61(\mathrm{~m}, 3 \mathrm{H})$ and 8.16-8.20 (m, 1H) ppm; ${ }^{13} \mathrm{C} \mathrm{NMR}\left(62.8 \mathrm{M} \mathrm{Hz}, \mathrm{CDCl}_{3}\right) \delta 9.52\left(\mathrm{CH}_{3}\right)$, $30.43\left(\mathrm{CH}_{2}\right), 33.77\left(\mathrm{CH}_{2}\right), 41.60\left(\mathrm{CH}_{2}\right), 47.19(\mathrm{CH}), 55.84\left(\mathrm{CH}_{3}, \mathrm{OMe}\right), 55.91\left(\mathrm{CH}_{3}, \mathrm{OMe}\right)$, $111.31(\mathrm{CH}), 112.19(\mathrm{CH}), 121.00(\mathrm{CH}), 125.71(\mathrm{C}), 126.82(\mathrm{CH}), 127.56(\mathrm{CH}), 128.78(\mathrm{CH})$, $131.14(\mathrm{C}), 133.62(\mathrm{CH}), 138.92(\mathrm{C}), 147.65(\mathrm{C}), 148.89(\mathrm{C}), 164.70(\mathrm{C}=\mathrm{O})$ and $173.56(\mathrm{C}=\mathrm{O})$ ppm; Found: M353.1627 (16.7\%) $\mathrm{C}_{21} \mathrm{H}_{23} \mathrm{NO}_{4}$ requires 353.1627. COSY and HETCOR experiments support the structure assignments.

Reaction of 2-benzyl-1,2,3,4-tetrahydro-1,3-isoquinolinedione with benzyl bromide and NaOH. A stirred solution of 2-benzyl-1,2,3,4-tetrahydro-1,3-isoquinolinedione $(1.0 \mathrm{~g}$, $4.0 \mathrm{mmol})$, sodium hydroxide $(0.17 \mathrm{~g}, 4.2 \mathrm{mmol})$ and benzyl bromide $(0.7 \mathrm{~g}, 4.1 \mathrm{mmol})$ in aqueous ethanol [1:1] (ca. $120 \mathrm{~mL}$ ) was heated under reflux for 3 hours and gave two products after flash chromatography eluting with ethyl acetate : light petroleum [1:4]. The first product 2,4,4-tribenzyl-1,2,3,4-tetrahydro-1,3-isoquinoline dione ${ }^{31}$ (0.29 g, 17\%), yellow oil : $\mathrm{v}_{\max } 3031$, 1710, 1667 and $1604 \mathrm{~cm}^{-1}$; ${ }^{1} \mathrm{H} \mathrm{NMR}\left(250 \mathrm{M} \mathrm{Hz} \mathrm{CDCl}_{3}\right) \delta 3.35\left(\mathrm{~d}, 2 \mathrm{H}, \mathrm{A}\right.$ of $\left.\mathrm{AB}, J_{\mathrm{AB}} 13.0\right), 3.78$ 
(d, 2H, B of AB, $\left.J_{\mathrm{AB}} 13.0\right), 4.89(\mathrm{~s}, 2 \mathrm{H}), 6.60-6.63(\mathrm{~m}, 4 \mathrm{H}), 6.87-7.40(\mathrm{~m}, 12 \mathrm{H}), 7.76-7.80(\mathrm{~m}$, 2H) and 7.95-7.98 (m, 1H) ppm; ${ }^{13} \mathrm{C}$ NMR (68.2 M Hz, CDCl 3$) 43.41\left(\mathrm{CH}_{2}\right), 48.48\left(2 \times \mathrm{CH}_{2}\right)$, $55.94(\mathrm{C}), 126.66(\mathrm{CH}), 126.86(2 \times \mathrm{CH}), 127.06(\mathrm{CH}), 127.48(\mathrm{CH}), 127.99(4 \times \mathrm{CH}), 128.23(2$ x CH), $128.47(2 \times \mathrm{CH}), 128.53(\mathrm{C}), 128.83(\mathrm{CH}), 129.42(4 \mathrm{x} \mathrm{CH}), 133.38(\mathrm{CH}), 135.40(2 \mathrm{x}$ C), $136.61(\mathrm{C}), 140.71(\mathrm{C}), 163.29(\mathrm{C}=\mathrm{O})$ and $174.44(\mathrm{C}=\mathrm{O}) \mathrm{ppm}$; Found: $\mathrm{M} 431.1888(37.0 \%)$ Calc. for $\mathrm{C}_{30} \mathrm{H}_{25} \mathrm{NO}_{2}$ 431.1885. The second product 2,4-dibenzyl-1,2,3,4-tetrahydro-1,3isoquinolinedione (7) $(0.57 \mathrm{~g}, 42 \%) \mathrm{mp} 98-100{ }^{\circ} \mathrm{C}$ from ethyl acetate : light petroleum: $\mathrm{v}_{\max }$ 1715,1669 and $1606 \mathrm{~cm}^{-1} ;{ }^{1} \mathrm{H}$ NMR $\left(400 \mathrm{M} \mathrm{Hz}, \mathrm{CDCl}_{3}\right) \delta 3.29$ (dd, $1 \mathrm{H}, \mathrm{A}$ of ABX, $J_{\mathrm{AB}} 13.2$, $\left.J_{\mathrm{AX}} 4.9\right), 3.42\left(\mathrm{dd}, 1 \mathrm{H}, \mathrm{B}\right.$ of $\left.\mathrm{ABX}, J_{\mathrm{AB}} 13.2, J_{\mathrm{BX}} 6.1\right), 4.25\left(\mathrm{dd}, 1 \mathrm{H}, \mathrm{X}\right.$ of $\left.\mathrm{ABX}, J_{\mathrm{AX}} 4.9, J_{\mathrm{BX}} 6.1\right)$, $5.06(\mathrm{~s}, 2 \mathrm{H}), 6.61-6.63(\mathrm{~m}, 2 \mathrm{H}), 7.01-7.59(\mathrm{~m}, 11 \mathrm{H})$ and $8.12-8.14(\mathrm{~m}, 1 \mathrm{H}) \mathrm{ppm} ;{ }^{13} \mathrm{C} \mathrm{NMR}(68.2$ $\left.\mathrm{M} \mathrm{Hz}, \mathrm{CDCl}_{3}\right) \delta 43.42\left(2 \times \mathrm{CH}_{2}\right), 48.16(\mathrm{CH}), 125.87(\mathrm{C}), 127.10(\mathrm{CH}), 127.28(\mathrm{CH}), 127.43$ $(\mathrm{CH}), 127.73(\mathrm{CH}), 128.17(2 \times \mathrm{CH}), 128.39(2 \times \mathrm{CH}), 128.86(\mathrm{CH}), 129.14(2 \times \mathrm{CH}), 129.28(2$ x CH), $133.36(\mathrm{CH}), 135.32(\mathrm{C}), 136.93(\mathrm{C}), 138.09(\mathrm{C}), 164.29(\mathrm{C}=\mathrm{O})$ and $173.00(\mathrm{C}=\mathrm{O}) \mathrm{ppm}$; Found: M341.1420 (29.0\%) $\mathrm{C}_{23} \mathrm{H}_{19} \mathrm{NO}_{2}$ requires 341.1416. COSY and HETCOR experiments support the structure assignments.

Reaction of 2-benzyl-1,2,3,4-tetrahydro-1,3-isoquinolinedione with isopropyl iodide and NaOH. A stirred solution of 2-benzyl-1,2,3,4-tetrahydro-1,3-isoquinolinedione (2.5 g, $10.0 \mathrm{mmol})$, sodium hydroxide $(0.44 \mathrm{~g}, 11.0 \mathrm{mmol})$ and isopropyl iodide $(1.7 \mathrm{~g}, 10.0 \mathrm{mmol})$ in aqueous ethanol [1:1] (ca. $140 \mathrm{~mL}$ ) was heated under reflux for 4 hours and gave after flash chromatography eluting with ethyl acetate : light petroleum [1:8] 2-benzyl-4-isopropyl-1,2,3,4tetrahydro-1,3-isoquinolinedione (1) $(1.23 \mathrm{~g}, 42 \%), \mathrm{mp} 72-74{ }^{\circ} \mathrm{C}$ from ethyl acetate : light petroleum: $\mathrm{v}_{\max } 2964,1715,1671$ and $1606 \mathrm{~cm}^{-1} ;{ }^{1} \mathrm{H}$ NMR $\left(400 \mathrm{M} \mathrm{Hz}, \mathrm{CDCl}_{3}\right) \delta 0.77(\mathrm{~d}, 3 \mathrm{H}, J$ 6.9), 0.91 (d, 3H, J 6.9), 2.23-2.31 (m, 1H), 3.79 (d, 1H, J4.2), 5.17 (q, 2H, AB, $\left.J_{\mathrm{AB}} 13.6\right), 7.25-$ $7.59(\mathrm{~m}, 8 \mathrm{H})$ and 8.22-8.24 (m, 1H) ppm; ${ }^{13} \mathrm{C} \mathrm{NMR}\left(100.5 \mathrm{M} \mathrm{Hz}, \mathrm{CDCl}_{3}\right) \delta 19.04\left(\mathrm{CH}_{3}\right), 19.77$ $\left(\mathrm{CH}_{3}\right), 37.61(\mathrm{CH}), 43.61\left(\mathrm{CH}_{2}\right), 53.38(\mathrm{CH}), 126.15(\mathrm{C}), 127.42(\mathrm{CH}), 127.50(\mathrm{CH}), 127.53$ $(\mathrm{CH}), 128.25(2 \times \mathrm{CH}), 128.65(\mathrm{CH}), 129.18(2 \times \mathrm{CH}), 133.05(\mathrm{CH}), 137.04(\mathrm{C}), 137.93(\mathrm{C})$, $165.45(\mathrm{C}=\mathrm{O})$ and $173.34(\mathrm{C}=\mathrm{O})$ ppm; Found: $\mathrm{M} 293.1410(32.6 \%) \mathrm{C}_{19} \mathrm{H}_{19} \mathrm{NO}_{2}$ requires 293.1416.

Reaction of 2-[2-(1H-3-Indolyl)ethyl]-1,2,3,4-tetrahydro-1,3-isoquinolinedione with isopropyl iodide ( 2 equivalents) and $\mathbf{N a O H}$. A stirred solution of 2-[2-(1H-3-indolyl)ethyl]1,2,3,4-tetrahydro-1,3-isoquinolinedione (11) (1.4 g, $4.6 \mathrm{mmol})$, sodium hydroxide (0.20 g, $5.0 \mathrm{mmol})$ and isopropyl iodide $(1.56 \mathrm{~g}, 9.2 \mathrm{mmol})$ in aqueous ethanol [1:1] (ca. $140 \mathrm{~mL})$ was heated under reflux for 3 hours and gave after flash chromatography eluting with ethyl acetate : light petroleum [1:3] 2-[2-(1H-3-indolyl)ethyl]-4-isopropyl-1,2,3,4-tetrahydro-1,3isoquinolinedione (14) $(0.70 \mathrm{~g}, 44 \%), \mathrm{mp} 135-137{ }^{\circ} \mathrm{C}$ from ethyl acetate : light petroleum: $\mathrm{v}_{\max }$ 3370 (broad), 3154, 1714, 1673 and $1606 \mathrm{~cm}^{-1} ;{ }^{1} \mathrm{H}$ NMR $\left(400 \mathrm{M} \mathrm{Hz}, \mathrm{CDCl}_{3}\right) \delta 0.92(\mathrm{~d}, 3 \mathrm{H}, J$ 6.9), 1.03 (d, 3H, J 6.8), 2.34-2.38 (m, 1H), 3.13-3.17 (m, 2H), 3.84 (d, 1H, J4.0), 4.21-4.28 (m, $1 \mathrm{H}), 4.36-4.44(\mathrm{~m}, 1 \mathrm{H}), 7.13-7.65(\mathrm{~m}, 7 \mathrm{H}) 7.94$ (d, 1H, J7.4), 8.25 (br.s, 1H, NH) and 8.28-8.30 (m, 1H) ppm; ${ }^{13} \mathrm{C}$ NMR (100.5 M Hz, $\left.\mathrm{CDCl}_{3}\right) \delta 19.24\left(\mathrm{CH}_{3}\right), 19.86\left(\mathrm{CH}_{3}\right), 24.37\left(\mathrm{CH}_{2}\right), 37.52$ $(\mathrm{CH}), 41.26\left(\mathrm{CH}_{2}\right), 53.42(\mathrm{CH}), 111.39(\mathrm{CH}), 113.11(\mathrm{C}), 119.56(\mathrm{CH}), 119.75(\mathrm{CH}), 122.30$ 
$(\mathrm{CH}), 122.50(\mathrm{CH}), 126.63(\mathrm{C}), 127.83(\mathrm{C}), 127.92(\mathrm{CH}), 127.96(\mathrm{CH}), 128.88(\mathrm{CH}), 133.38$ $(\mathrm{CH}), 136.60(\mathrm{C}), 138.35(\mathrm{C}), 165.55(\mathrm{C}=\mathrm{O})$ and $173.50(\mathrm{C}=\mathrm{O})$ ppm; Found: $\mathrm{M} 346.1683(7.9 \%)$ $\mathrm{C}_{22} \mathrm{H}_{22} \mathrm{~N}_{2} \mathrm{O}_{2}$ requires 346.1681 .

\section{The oxidation of 4-monosubstituted-1,2,3,4-tetrahydro-1,3-isoquinoline-diones. General method}

A magnetically stirred solution of the particular 1,3-isoquinolinedione, sodium hydroxide (1 equivalent or catalytic) in a given volume of oxygen-saturated ethanol was heated under reflux for a given length of time. Subsequently, the reaction mixture was allowed to cool to room temperature. The reaction was then adjusted to neutral $\mathrm{pH}$ using diluted hydrochloric acid. Then DCM $(150 \mathrm{~mL})$ and water $(150 \mathrm{~mL})$ were added to the reaction mixture followed by vigorous shaking. The organic layer was then collected and the aqueous layer was further extracted with DCM $(100 \mathrm{~mL})$. The combined organic extracts were dried over magnesium sulfate, concentrated in vacuo and the residue was purified by flash chromatography on silica gel and/or recrystallisation. Oxygen-saturation of ethanol was carried out by bubbling oxygen gas through the particular volume of ethanol for 5-10 minutes prior to dissolving the particular 1,3isoquinolinedione. The following oxidation reactions were conducted:

Oxidation of 2-benzyl-4-ethyl-1,2,3,4-tetrahydro-1,3-isoquinolinedione. A stirred solution of 2-benzyl-4-ethyl-1,2,3,4-tetrahydro-1,3-isoquinolinedione (4) and sodium hydroxide in oxygenated ethanol was heated under reflux for the stated period and gave two products after flash chromatography eluting with ethyl acetate : light petroleum [1:4] (reaction conditions and yields are listed in Table Exp. 1). The first product 2-benzyl-4-ethyl-4-hydroxy-1,2,3,4tetrahydro-1,3-isoquinolinedione (5), oil : $\mathrm{v}_{\max } 3464$ (broad), 2972, 1722, 1674 and $1606 \mathrm{~cm}^{-1}$; ${ }^{1} \mathrm{H}$ NMR (400 M Hz, CDCl $) \delta 0.65$ (t, 3H, J 7.5), 1.77-1.93 (m, 2H), 3.85 (br.s, 1H, OH), 5.14 $\left(\mathrm{d}, 1 \mathrm{H}, \mathrm{A}\right.$ of $\left.\mathrm{AB}, J_{\mathrm{AB}} 13.9\right), 5.19\left(\mathrm{~d}, 1 \mathrm{H}, J_{\mathrm{AB}} 13.9\right), 7.28-7.72(\mathrm{~m}, 8 \mathrm{H})$ and $8.18-8.20(\mathrm{~m}, 1 \mathrm{H})$ ppm; ${ }^{13} \mathrm{C}$ NMR $\left(100.5 \mathrm{M} \mathrm{Hz}, \mathrm{CDCl}_{3}\right) \delta 8.06\left(\mathrm{CH}_{3}\right), 40.70\left(\mathrm{CH}_{2}\right), 44.43\left(\mathrm{CH}_{2}\right), 75.77(\mathrm{C}), 124.71$ (C), $125.51(\mathrm{CH}), 128.12(\mathrm{CH}), 128.73(\mathrm{CH}), 128.88(2 \times \mathrm{CH}), 129.03(\mathrm{CH}), 129.35(2 \times \mathrm{CH})$, $134.37(\mathrm{CH}), 136.97(\mathrm{C}), 141.22(\mathrm{C}), 164.37(\mathrm{C}=\mathrm{O})$ and $177.36(\mathrm{C}=\mathrm{O}) \mathrm{ppm}$; Found: M295.1211 (14.5\%) $\mathrm{C}_{18} \mathrm{H}_{17} \mathrm{NO}_{3}$ requires 295.1208. The second product $N$-1-benzyl-1-ethyl-3-oxo-1,3dihydro-1-isobenzofurancarboxamide (6), $\mathrm{mp} 127-129{ }^{\circ} \mathrm{C}$ from ethyl acetate : light petroleum: $\mathrm{v}_{\max } 3365$ (broad), 2974, 1772, 1675 and $1600 \mathrm{~cm}^{-1} ;{ }^{1} \mathrm{H} \mathrm{NMR}\left(400 \mathrm{M} \mathrm{Hz}, \mathrm{CDCl}_{3}\right) \delta 0.88(\mathrm{t}, 3 \mathrm{H}$, J 7.4), 2.01-2.10 (m, 1H), 2.46-2.55 (m, 1H), 4.25 (dd, 1H, J5.2, 14.7), 4.56 (dd, 1H, J6.6, 14.7), 7.10 (br.s, $1 \mathrm{H}, \mathrm{N}-\mathrm{H}), 7.22-7.31(\mathrm{~m}, 5 \mathrm{H}), 7.56-7.59(\mathrm{~m}, 1 \mathrm{H}), 7.71-7.75(\mathrm{~m}, 1 \mathrm{H})$ and 7.83-7.90 (m, $2 \mathrm{H}) \mathrm{ppm} ;{ }^{13} \mathrm{C} \mathrm{NMR}\left(100.5 \mathrm{M} \mathrm{Hz}, \mathrm{CDCl}_{3}\right) \delta 8.33\left(\mathrm{CH}_{3}\right), 32.09\left(\mathrm{CH}_{2}\right), 43.93\left(\mathrm{CH}_{2}\right), 89.88(\mathrm{C})$, $123.91(\mathrm{CH}), 124.61(\mathrm{C}), 125.88(\mathrm{CH}), 128.06(\mathrm{CH}), 128.14(2 \times \mathrm{CH}), 129.12(2 \times \mathrm{CH}), 130.24$ $(\mathrm{CH}), 135.31(\mathrm{CH}), 137.77(\mathrm{C}), 149.36(\mathrm{C}), 169.25(\mathrm{C}=\mathrm{O})$ and $169.58(\mathrm{C}=\mathrm{O})$ ppm; Found: $\mathrm{C}$, $72.55, \mathrm{H}, 5.75, \mathrm{~N}, 4.77 \% \mathrm{C}_{18} \mathrm{H}_{17} \mathrm{NO}_{3}$ requires $\mathrm{C}, 73.22, \mathrm{H}, 5.76, \mathrm{~N}, 4.74 \%$; Found: $\mathrm{M} 295.1210$ (22.8\%) $\mathrm{C}_{18} \mathrm{H}_{17} \mathrm{NO}_{3}$ requires 295.1208 . 
Table 1. Exp. 1

\begin{tabular}{cccccccccccc}
\hline & $\begin{array}{c}\text { Starting } \\
\text { Material }\end{array}$ & $\mathrm{NaOH}$ & Reaction & Solvent & \multicolumn{3}{c}{ Product yields } \\
\hline No & $\mathrm{mg}$ & $\mathrm{mmol}$ & $\mathrm{mg}$ & $\mathrm{mmol}$ & $\begin{array}{c}\text { Time } \\
\text { (hours) }\end{array}$ & $\begin{array}{c}\text { Volume } \\
(\mathrm{mL} .)\end{array}$ & $\mathbf{5 , m g}$ & $\mathbf{5 , \%}$ & $\mathbf{6 , m g}$ & $\mathbf{6 , \%} \%$ \\
\hline 1 & 210 & 0.75 & 33 & 0.83 & 4 & 70 & 22 & 10 & 113 & 51 \\
2 & 210 & 0.75 & 5 & 0.11 & 9 & 70 & 80 & 36 & 55 & 25 \\
\hline
\end{tabular}

Oxidation of 2-(3,4-dimethoxyphenethyl)-4-ethyl-1,2,3,4-tetrahydro-1,3-isoquinolinedione. A stirred solution of 2-(3,4-dimethoxyphenethyl)-4-ethyl-1,2,3,4-tetrahydro-1,3isoquinolinedione (12) (1.0 g, $2.83 \mathrm{mmol})$ and sodium hydroxide $(0.11 \mathrm{~g}, 2.83 \mathrm{mmol})$ in oxygenated ethanol $(70 \mathrm{~mL})$ was heated under reflux for 24 hours and gave two products after flash chromatography eluting with ethyl acetate : light petroleum [1:4]. The first product 2-(3,4dimethoxyphenethyl)-4-ethyl-4-hydroxy-1,2,3,4-tetrahydro-1,3-isoquinolinedione (15) (0.10 g, 10\%), oil : $\mathrm{v}_{\max } 3464$ (broad), 2967, 1718, 1671 and $1606 \mathrm{~cm}^{-1} ;{ }^{1} \mathrm{H} \mathrm{NMR}\left(250 \mathrm{M} \mathrm{Hz}, \mathrm{CDCl}_{3}\right) \delta$ $0.70(\mathrm{t}, 3 \mathrm{H}, J$ 7.5), 1.75-1.88 (m, 2H), 2.88 (t, 2H, J 7.8), 3.76 (br.s, $1 \mathrm{H}, \mathrm{OH}), 3.85\left(3 \mathrm{H}, \mathrm{CH}_{3}\right.$, $\mathrm{OMe}), 3.86\left(3 \mathrm{H}, \mathrm{CH}_{3}, \mathrm{OMe}\right), 4.08-4.17(\mathrm{~m}, 1 \mathrm{H}), 4.23-4.32(\mathrm{~m}, 1 \mathrm{H}), 6.78-6.83(\mathrm{~m}, 3 \mathrm{H}), 7.46-$ $7.53(\mathrm{~m}, 1 \mathrm{H}), 7.67-7.70(\mathrm{~m}, 2 \mathrm{H})$ and 8.13-8.16 (m, 1H) ppm; ${ }^{13} \mathrm{C} \mathrm{NMR}\left(62.8 \mathrm{M} \mathrm{Hz}, \mathrm{CDCl}_{3}\right) \delta$ $7.87\left(\mathrm{CH}_{3}\right), 33.78\left(\mathrm{CH}_{2}\right), 40.22\left(\mathrm{CH}_{2}\right), 42.21\left(\mathrm{CH}_{2}\right), 55.78\left(\mathrm{CH}_{3}, \mathrm{MeO}\right), 55.93\left(\mathrm{CH}_{3}, \mathrm{MeO}\right)$, $75.25(\mathrm{C}), 111.33(\mathrm{CH}), 112.14(\mathrm{CH}), 121.00(\mathrm{CH}), 124.63(\mathrm{C}), 125.18(\mathrm{CH}), 128.34(\mathrm{CH})$, $128.43(\mathrm{CH}), 130.70(\mathrm{C}), 133.93(\mathrm{CH}), 140.81(\mathrm{C}), 147.78(\mathrm{C}), 148.94(\mathrm{C}), 163.98(\mathrm{C}=\mathrm{O})$ and $177.01(\mathrm{C}=\mathrm{O})$ ppm; Found: $\mathrm{M} 369.1574(4.5 \%) \mathrm{C}_{21} \mathrm{H}_{23} \mathrm{NO}_{5}$ requires 369.1576. The second product N1-(3,4-dimethoxyphenethyl)-1-ethyl-3-oxo-1,3-dihydro-1-isobenzofuran carboxamide (16) $\left(0.53 \mathrm{~g}, 51 \%\right.$ ), oil : $\mathrm{v}_{\max } 3369$ (broad), 2938, 1771, 1674 and $1592 \mathrm{~cm}^{-1}$; ${ }^{1} \mathrm{H}$ NMR (400 M $\left.\mathrm{Hz}, \mathrm{CDCl}_{3}\right) \delta 0.84(\mathrm{t}, 3 \mathrm{H}, J$ 7.4), 1.98-2.05 (m, 1H), 2.39-2.45 (m, 1H), $2.72(\mathrm{t}, 2 \mathrm{H}, J$ 7.0), 3.423.57 (m, 2H), 3.84 (s, 3H, OMe), 3.85 (s, 3H, OMe), 6.60-6.75 (m, 4H), 7.54-7.58 (m, 1H), 7.69$7.73(\mathrm{~m}, 1 \mathrm{H})$ and 7.82-7.85 (m, 2H) ppm; ${ }^{13} \mathrm{C} \mathrm{NMR}\left(100.5 \mathrm{M} \mathrm{Hz}, \mathrm{CDCl}_{3}\right) \delta 8.25\left(\mathrm{CH}_{3}\right), 31.82$ $\left(\mathrm{CH}_{2}\right), 35.54\left(\mathrm{CH}_{2}\right), 41.09\left(\mathrm{CH}_{2}\right), 56.18\left(\mathrm{CH}_{3}, \mathrm{OMe}\right), 56.29\left(\mathrm{CH}_{3}, \mathrm{OMe}\right), 89.83(\mathrm{C}), 111.86$ $(\mathrm{CH}), 112.22(\mathrm{CH}), 121.00(\mathrm{CH}), 123.85(\mathrm{CH}), 124.52(\mathrm{C}), 125.76(\mathrm{CH}), 130.16(\mathrm{CH}), 131.08$ (C), $135.22(\mathrm{CH}), 148.18(\mathrm{C}), 149.37(\mathrm{C}), 149.46(\mathrm{C}), 169.22(\mathrm{C}=\mathrm{O})$ and $169.49(\mathrm{C}=\mathrm{O}) \mathrm{ppm}$; Found: $\mathrm{M} 369.1573(32.7 \%) \mathrm{C}_{21} \mathrm{H}_{23} \mathrm{NO}_{5}$ requires 369.1576 .

Oxidation of 2,4-dibenzyl-1,2,3,4-tetrahydro-1,3-isoquinolinedione. A stirred solution of 2,4dibenzyl-1,2,3,4-tetrahydro-1,3-isoquinolinedione (7) and sodium hydroxide in oxygenated ethanol was heated under reflux for the stated period and gave two products after flash chromatography eluting with ethyl acetate : light petroleum [1:7] (reaction conditions and yields are listed in Table Exp. 2). The first product 2-dibenzyl-4-hydroxy-1,2,3,4-tetrahydro-1,3isoquinolinedione (8), $\mathrm{mp} 109-111^{\circ} \mathrm{C}$ ether: $\mathrm{v}_{\max } 3459$ (broad), 3032, 1719, 1673 and $1605 \mathrm{~cm}^{-1}$; ${ }^{1} \mathrm{H}$ NMR $\left(400 \mathrm{M} \mathrm{Hz}, \mathrm{CDCl}_{3}\right) \delta 3.09\left(\mathrm{~d}, 1 \mathrm{H}, \mathrm{A}\right.$ of AB, $\left.J_{\mathrm{AB}} 12.9\right), 3.19\left(\mathrm{~d}, 1 \mathrm{H}, \mathrm{B}\right.$ of $\left.\mathrm{AB}, J_{\mathrm{AB}} 12.9\right)$, 3.95 (br.s, $1 \mathrm{H}, \mathrm{OH}), 4.96\left(\mathrm{~d}, 1 \mathrm{H}, \mathrm{A}\right.$ of $\left.\mathrm{AB}, J_{\mathrm{AB}} 13.8\right), 5.08\left(\mathrm{~d}, 1 \mathrm{H}, J_{\mathrm{AB}} 13.8\right), 6.51-6.52(\mathrm{~m}, 2 \mathrm{H})$, 7.04-7.70 (m, 11H) and 8.08-8.10 (m, 1H) ppm; ${ }^{13} \mathrm{C} \mathrm{NMR}\left(100.5 \mathrm{M} \mathrm{Hz}, \mathrm{CDCl}_{3}\right) \delta 44.50\left(\mathrm{CH}_{2}\right)$, 
$54.11\left(\mathrm{CH}_{2}\right), 76.18(\mathrm{C}), 125.17(\mathrm{C}), 125.78(\mathrm{CH}), 127.86(\mathrm{CH}), 128.16(\mathrm{CH}), 128.41(2 \times \mathrm{CH})$, $128.61(\mathrm{CH}), 128.90(2 \times \mathrm{CH}), 128.94(\mathrm{CH}), 129.82(2 \times \mathrm{CH}), 130.38(2 \times \mathrm{CH}), 133.45(\mathrm{C})$, $134.37(\mathrm{CH}), 136.75(\mathrm{C}), 140.25(\mathrm{C}), 163.76(\mathrm{C}=\mathrm{O})$ and $176.43(\mathrm{C}=\mathrm{O})$ ppm; Found: $\mathrm{M} 357.1365$ $(11.5 \%) \mathrm{C}_{23} \mathrm{H}_{19} \mathrm{NO}_{3}$ requires 357.1365. COSY experiment supports the structure assignments. The second product N1,1-dibenzyl-3-oxo-1,3-dihydro-1-isobenzofurancarboxamide (9), mp 96$98{ }^{\circ} \mathrm{C}$ from ethyl acetate : light petroleum: $\mathrm{v}_{\max } 3364$ (broad), 3031, 1774, 1676 and $1600 \mathrm{~cm}^{-1}$; ${ }^{1} \mathrm{H}$ NMR (400 M Hz, $\left.\mathrm{CDCl}_{3}\right) \delta 3.26\left(\mathrm{~d}, 1 \mathrm{H}, \mathrm{A}\right.$ of $\left.\mathrm{AB}, J_{\mathrm{AB}} 14.0\right), 3.75\left(\mathrm{~d}, 1 \mathrm{H}, \mathrm{B}\right.$ of $\left.\mathrm{AB}, J_{\mathrm{AB}} 14.0\right)$, 4.10 (dd, 1H, J4.7, 14.7), 4.50 (dd, 1H, J6.9, 14.7), 6.70 (br.s, 1H, N-H), 6.95-6.97 (m, 2H), 7.22-7.30 (m, 8H), 7.58-7.60 (m, 1H), 7.79-7.81 (m, 2H) and 8.06-8.08 (m, 1H) ppm; ${ }^{13} \mathrm{C}$ NMR $\left(100.5 \mathrm{M} \mathrm{Hz}, \mathrm{CDCl}_{3}\right) \delta 43.83\left(\mathrm{CH}_{2}\right), 44.88\left(\mathrm{CH}_{2}\right), 89.17(\mathrm{C}), 124.23(\mathrm{CH}), 124.68(\mathrm{C}), 125.92$ $(\mathrm{CH}), 127.72(\mathrm{CH}), 128.00(2 \times \mathrm{CH}), 128.66(2 \times \mathrm{CH}), 129.05(\mathrm{CH}), 130.37(\mathrm{CH}), 130.91(2 \times$ $\mathrm{CH}), 133.96(\mathrm{C}), 135.26(\mathrm{CH}), 137.23(\mathrm{C}), 149.00(\mathrm{C}), 168.51(\mathrm{C}=\mathrm{O})$ and $169.10(\mathrm{C}=\mathrm{O}) \mathrm{ppm}$; Found: $\mathrm{M} 357.1366(4.1 \%) \mathrm{C}_{23} \mathrm{H}_{19} \mathrm{NO}_{3}$ requires 357.1365. COSY experiment supports the structure assignments.

Table 2. Exp. 2

\begin{tabular}{|c|c|c|c|c|c|c|c|c|c|}
\hline \multicolumn{2}{|l|}{$\begin{array}{l}\text { Starting } \\
\text { Material }\end{array}$} & \multicolumn{2}{|l|}{$\mathrm{NaOH}$} & \multirow{2}{*}{$\begin{array}{l}\text { Reaction } \\
\text { Time } \\
\text { (hours) }\end{array}$} & \multirow{2}{*}{$\begin{array}{l}\text { Solvent } \\
\text { Volume } \\
(\mathrm{mL} .)\end{array}$} & \multicolumn{4}{|c|}{ Product yields } \\
\hline $\mathrm{mg}$ & mmol & $\mathrm{mg}$ & $\mathrm{mmol}$ & & & $\begin{array}{l}6.5 \\
\mathrm{mg}\end{array}$ & $\%$ & $\begin{array}{l}\mathbf{6 . 6} \\
\mathrm{mg}\end{array}$ & $\%$ \\
\hline 230 & 0.67 & 4 & 0.10 & 17 & 70 & 80 & 33 & 30 & 13 \\
\hline
\end{tabular}

\section{Oxidation of 2-benzyl-4-isopropyl-1,2,3,4-tetrahydro-1,3-isoquinolinedione}

Procedure A. A stirred solution of 2-benzyl-4-isopropyl-1,2,3,4-tetrahydro-1,3-isoquinoline dione (1) $(0.20 \mathrm{~g}, 0.68 \mathrm{mmol})$, sodium hydroxide $(4.1 \mathrm{mg}, 0.10 \mathrm{mmol})$ and triethyl phosphite $(0.34 \mathrm{~g}, 2.04 \mathrm{mmol})$ in ethanol $(70 \mathrm{~mL})$ was bubbled with oxygen gas over 18 hours at room temperature. The reaction was subsequently terminated by adding DCM $(150 \mathrm{~mL})$ and water $(150 \mathrm{~mL})$ followed by vigorous shaking. The organic layer was then collected and the aqueous layer was further extracted with DCM $(100 \mathrm{~mL})$. The combined organic extracts were dried over magnesium sulfate, concentrated in vacuo and gave after flash chromatography eluting with ethyl acetate : light petroleum [1:4] 2-benzyl-4-hydroxy-4-isopropyl-1,2,3,4-tetrahydro-1,3isoquinolinedione (3) (0.20 g, 97\%), oil : $\mathrm{v}_{\max } 3482$ (broad), 2969, 1719, 1673 and $1605 \mathrm{~cm}^{-1} ;{ }^{1} \mathrm{H}$ NMR (400 M Hz, $\left.\mathrm{CDCl}_{3}\right) \delta 0.68$ (d, 3H, J 6.7), 0.73 (d, 3H, J 6.7), 1.87 (h, 1H, J 6.7), 3.83 (br.s, $1 \mathrm{H}, \mathrm{OH}), 5.12\left(\mathrm{~d}, 1 \mathrm{H}, \mathrm{A}\right.$ of $\left.\mathrm{AB}, J_{\mathrm{AB}} 13.8\right), 5.19\left(\mathrm{~d}, 1 \mathrm{H}, J_{\mathrm{AB}} 13.8\right), 7.30-7.70(\mathrm{~m}, 8 \mathrm{H})$ and 8.17$8.19(\mathrm{~m}, 1 \mathrm{H}) \mathrm{ppm} ;{ }^{13} \mathrm{C} \mathrm{NMR}\left(100.5 \mathrm{M} \mathrm{Hz}, \mathrm{CDCl}_{3}\right) \delta 16.78\left(\mathrm{CH}_{3}\right), 17.04\left(\mathrm{CH}_{3}\right), 42.99(\mathrm{CH})$, $44.52\left(\mathrm{CH}_{2}\right), 78.03(\mathrm{C}), 125.45(\mathrm{C}), 126.34(\mathrm{CH}), 128.18(\mathrm{CH}), 128.60(\mathrm{CH}), 128.71(\mathrm{CH})$, $128.81(2 \times \mathrm{CH}), 129.65(2 \times \mathrm{CH}), 133.62(\mathrm{CH}), 136.83(\mathrm{C}), 140.26(\mathrm{C}), 164.77(\mathrm{C}=\mathrm{O})$ and $177.31(\mathrm{C}=\mathrm{O})$ ppm; Found: $\mathrm{M} 309.1366(6.0 \%) \mathrm{C}_{19} \mathrm{H}_{19} \mathrm{NO}_{3}$ requires 309.1365 .

Procedure B. Using the general method, a stirred solution of 2-benzyl-4-isopropyl-1,2,3,4tetrahydro-1,3-isoquinolinedione (1) and sodium hydroxide in oxygenated ethanol (aqueous or 
anhydrous) was heated under reflux for a given period of time and gave a product after flash chromatography eluting with ethyl acetate : light petroleum [1:6] (reaction conditions and yields are listed in Table Exp. 3.) N1-benzyl-1-isopropyl-3-oxo-1,3-dihydro-1isobenzofurancarboxamide (2), $\mathrm{mp} 143-145{ }^{\circ} \mathrm{C}$ from ethyl acetate : light petroleum: $\mathrm{v}_{\max } 3366$ (broad), 2971, 1773, 1676 and $1598 \mathrm{~cm}^{-1} ;{ }^{1} \mathrm{H} \mathrm{NMR}\left(400 \mathrm{M} \mathrm{Hz}, \mathrm{CDCl}_{3}\right) \delta 0.55$ (d, 3H, J 6.8), 1.13 (d, 3H, J 6.8), 2.83 (h, 1H, J 6.8), 4.22 (dd, 1H, J5.1, 14.7), 4.57 (dd, 1H, J6.6, 14.7), 7.06 (br.s, $1 \mathrm{H}, \mathrm{N}-\mathrm{H}), 7.22-7.31(\mathrm{~m}, 5 \mathrm{H}), 7.56-7.58(\mathrm{~m}, 1 \mathrm{H}), 7.71-7.74(\mathrm{~m}, 1 \mathrm{H})$ and $7.83-7.88(\mathrm{~m}, 2 \mathrm{H})$ ppm; ${ }^{13} \mathrm{C}$ NMR $\left(100.5 \mathrm{M} \mathrm{Hz}, \mathrm{CDCl}_{3}\right) \delta 15.35\left(\mathrm{CH}_{3}\right), 17.76\left(\mathrm{CH}_{3}\right), 35.52(\mathrm{CH}), 43.77\left(\mathrm{CH}_{2}\right)$, $92.06(\mathrm{C}), 123.71(\mathrm{CH}), 124.61(\mathrm{C}), 125.65(\mathrm{CH}), 128.04(\mathrm{CH}), 128.22(2 \mathrm{x} \mathrm{CH}), 128.96(2 \mathrm{x}$ $\mathrm{CH}), 130.02(\mathrm{CH}), 135.16(\mathrm{CH}), 137.60(\mathrm{C}), 149.06(\mathrm{C}), 169.30(\mathrm{C}=\mathrm{O})$ and $169.76(\mathrm{C}=\mathrm{O}) \mathrm{ppm}$; Found: $\mathrm{M} 309.1363(12.2 \%) \mathrm{C}_{19} \mathrm{H}_{19} \mathrm{NO}_{3}$ requires 309.1365. HETCOR and COSY experiments support the structure assignments.

Table 3. Exp. 3

\begin{tabular}{|c|c|c|c|c|c|c|c|c|c|}
\hline \multirow{3}{*}{ No } & \multicolumn{2}{|c|}{$\begin{array}{l}\text { Starting } \\
\text { Material }\end{array}$} & \multicolumn{2}{|c|}{$\mathrm{NaOH}$} & \multirow{3}{*}{$\begin{array}{l}\text { Reaction } \\
\text { Time } \\
\text { (hours) }\end{array}$} & \multirow{3}{*}{$\begin{array}{l}\text { Solvent } \\
\text { Volume } \\
\text { (mL.) }\end{array}$} & \multicolumn{3}{|c|}{ Product yields } \\
\hline & \multirow{2}{*}{$\mathrm{mg}$} & \multirow{2}{*}{ mmol } & \multirow{2}{*}{$\mathrm{mg}$} & \multirow{2}{*}{ mmol } & & & & \multicolumn{2}{|l|}{2} \\
\hline & & & & & & & & $\mathrm{mg}$ & $\%$ \\
\hline 1 & 620 & 2.10 & 92 & 2.30 & 6 & $65^{*}$ & 0 & 140 & 22 \\
\hline 2 & 550 & 1.90 & 83 & 2.10 & 7 & 70 & 0 & 600 & $>99$. \\
\hline 3 & 230 & 0.74 & 0 & 0 & 5 & 50 & 0 & 0 & 0 \\
\hline 4 & 380 & 1.30 & 8 & 0.20 & 5 & 50 & 0 & 358 & 89 \\
\hline 5 & 230 & 0.74 & 30 & 0.75 & 5 & 60 & 0 & 240 & $>99$. \\
\hline
\end{tabular}

* Solvent: ethanol/water [5:8]

Oxidation of 2-[2-(1H-3-indolyl)ethyl]-4-isopropyl-1,2,3,4-tetrahydro-1,3-isoquinolinedione. A stirred solution 2-[2-(1H-3-indolyl)ethyl]-4-isopropyl-1,2,3,4-tetrahydro-1,3isoquinolinedione (14) $(0.20 \mathrm{~g}, 0.58 \mathrm{mmol})$ and sodium hydroxide $(25 \mathrm{mg}, 0.63 \mathrm{mmol})$ in oxygenated ethanol $(40 \mathrm{~mL})$ was heated under reflux for 5 hours and gave N1-[2-(1H-3indolyl)ethyl]-1-isopropyl-3-oxo-1,3-dihydro-1-isobenzofurancarboxamide (17) (0.19 g, 91\%), mp 187-189 ${ }^{\circ} \mathrm{C}$ from ethyl acetate : light petroleum: $\mathrm{v}_{\max } 3406$ (broad), 2970, 1771 and $1669 \mathrm{~cm}^{-}$ 1; ${ }^{1} \mathrm{H}$ NMR (400 M Hz, $\left.\mathrm{CDCl}_{3}\right) \delta 0.56(\mathrm{~d}, 3 \mathrm{H}, J$ 6.8), 1.11 (d, 3H, J 6.8), 2.81 (h, 1H, J 6.8), 2.94 (t, 2H, J 6.9), 3.50-3.69 (m, 2H), 6.72 (br.s, 1H, N-H), 6.91 (d, 1H, J 2.0), 7.10-7.13 (m, 1H), 7.19-7.23 (m, 1H), 7.36-7.38 (m, 1H), 7.55-7.60 (m, 2H), 7.71-7.75 (m, 1H), 7.86-7.88 (m, 2H) and 8.17 (br.s, $1 \mathrm{H}, \mathrm{N}-\mathrm{H}) \mathrm{ppm} ;{ }^{13} \mathrm{C} \mathrm{NMR}\left(100.5 \mathrm{M} \mathrm{Hz}, \mathrm{CDCl}_{3}\right) \delta 15.49\left(\mathrm{CH}_{3}\right), 17.89\left(\mathrm{CH}_{3}\right)$, $25.70\left(\mathrm{CH}_{2}\right), 35.48(\mathrm{CH}), 40.00\left(\mathrm{CH}_{2}\right), 92.29(\mathrm{C}), 111.67(\mathrm{CH}), 112.77(\mathrm{C}), 118.92(\mathrm{CH}), 119.91$ $(\mathrm{CH}), 122.37(\mathrm{CH}), 122.59(\mathrm{CH}), 123.89(\mathrm{CH}), 124.75(\mathrm{C}), 125.75(\mathrm{CH}), 127.44(\mathrm{C}), 130.12$ $(\mathrm{CH}), 135.30(\mathrm{CH}), 136.82(\mathrm{C}), 149.30(\mathrm{C}), 169.38(\mathrm{C}=\mathrm{O})$ and $169.95(\mathrm{C}=\mathrm{O})$ ppm; Found: $\mathrm{M}$ 
362.1636 (14.0\%) $\mathrm{C}_{22} \mathrm{H}_{22} \mathrm{~N}_{2} \mathrm{O}_{3}$ requires 362.1630. HETCOR and COSY experiments support the structure assignments.

\section{Bischler-Napieralski cyclisation of isobenzofuran carboxamides. General method}

A magnetically stirred solution of the particular isobenzofuran carboxamide and phosphoryl chloride (5-10 equivalents) in a given volume of dry acetonitrile was heated under reflux for a given length of time. Subsequently, the reaction mixture was allowed to cool to room temperature. The reaction mixture was then quenched carefully with dilute aqueous ammonia solution. Then DCM $(100 \mathrm{~mL})$ and water $(100 \mathrm{~mL})$ were added to the reaction mixture followed by vigorous shaking. The organic layer was then collected and the aqueous layer was further extracted with DCM $(100 \mathrm{~mL})$. The combined organic extracts were dried over magnesium sulfate, concentrated in vacuo and the residue was purified by flash chromatography on silica gel and/or recrystallisation.

3-(6,7-Dimethoxy-3,4-dihydro-1-isoquinolinyl)-3-ethyl-1,3-dihydro-1-isobenzofuranone. A stirred solution of N1-(3,4-dimethoxyphenethyl)-1-ethyl-3-oxo-1,3-dihydro-1-isobenzofuran carboxamide (16) (0.49 g, $1.32 \mathrm{mmol})$ and phosphoryl chloride (1.0 g, $6.54 \mathrm{mmol})$ in dry acetonitrile $(10 \mathrm{~mL})$ was heated under reflux for 4 hours and 20 minutes and gave the title compound (18) after flash chromatography eluting with ethyl acetate : light petroleum [1:5] (0.27 g, 58\%), oil : $\mathrm{v}_{\max } 2939,1765$ and $1606 \mathrm{~cm}^{-1} ;{ }^{1} \mathrm{H}$ NMR $\left(400 \mathrm{M} \mathrm{Hz}, \mathrm{CDCl}_{3}\right) \delta 0.75(\mathrm{t}, 3 \mathrm{H}, J$ 7.5), 2.22-2.27 (m, 1H), 2.51-2.60 (m, 3H), 3.58-3.62 (m, 1H), 3.70-3.75 (m, 1H), $3.85(\mathrm{~s}, 3 \mathrm{H}$, $\mathrm{OMe}), 3.88(\mathrm{~s}, 3 \mathrm{H}, \mathrm{OMe}), 6.66(\mathrm{~s}, 1 \mathrm{H}), 7.39(\mathrm{~s}, 1 \mathrm{H}), 7.49-7.53(\mathrm{~m}, 1 \mathrm{H}), 7.66-7.67(\mathrm{~m}, 2 \mathrm{H})$ and 7.86-7.89 (m, 1H) ppm; ${ }^{13} \mathrm{C} \mathrm{NMR}\left(100.5 \mathrm{M} \mathrm{Hz}, \mathrm{CDCl}_{3}\right) \delta 7.91\left(\mathrm{CH}_{3}\right), 26.54\left(\mathrm{CH}_{2}\right), 32.89\left(\mathrm{CH}_{2}\right)$, $47.72\left(\mathrm{CH}_{2}\right), 56.20\left(\mathrm{CH}_{3}, \mathrm{MeO}\right), 56.46\left(\mathrm{CH}_{3}, \mathrm{MeO}\right), 93.28(\mathrm{C}), 110.49(\mathrm{CH}), 110.75(\mathrm{CH})$, $120.22(\mathrm{C}), 124.96(\mathrm{CH}), 125.40(\mathrm{CH}), 126.20(\mathrm{C}), 129.47(\mathrm{CH}), 133.23(\mathrm{C}), 134.14(\mathrm{CH})$, $147.47(\mathrm{C}), 151.01(\mathrm{C}), 151.89(\mathrm{C}), 164.53(\mathrm{C}=\mathrm{N})$ and $170.64(\mathrm{C}=\mathrm{O})$ ppm; Found: $\mathrm{M} 351.1468$ $(80.5 \%) \mathrm{C}_{21} \mathrm{H}_{21} \mathrm{NO}_{4}$ requires 351.1471 . COSY experiment supports the structural assignment.

3-(4,9-Dihydro-3H- $\beta$-carbolin-1-yl)-3-isopropyl-1,3-dihydro-1-isobenzofuranone. A stirred solution of N1-[2-(1H-3-indolyl)ethyl]-1-isopropyl-3-oxo-1,3-dihydro-1-isobenzofuran carboxamide (17) $(0.15 \mathrm{~g}, 0.41 \mathrm{mmol})$ and phosphoryl chloride $(0.61 \mathrm{~g}, 4.0 \mathrm{mmol})$ in dry acetonitrile $(5 \mathrm{~mL})$ was heated under reflux for 3 hours and 30 minutes and gave the title compound (19) after flash chromatography eluting with ethyl acetate : light petroleum [1:4] (31mg, 22\%), mp 148-1; ${ }^{1} \mathrm{H}$ NMR (400 M Hz, $\mathrm{CDCl}_{3}$ ) $\delta 0.77$ (d, 3H, J 6.8), 1.04 (d, 3H, J 6.8), 2.84-7.96 (m, 8H) and 9.20 (br.s, $1 \mathrm{H}, \mathrm{N}-\mathrm{H}) \mathrm{ppm} ;{ }^{13} \mathrm{C} \mathrm{NMR}\left(100.5 \mathrm{M} \mathrm{Hz}, \mathrm{CDCl}_{3}\right) \delta 16.43\left(\mathrm{CH}_{3}\right)$, $17.56\left(\mathrm{CH}_{3}\right), 19.40\left(\mathrm{CH}_{2}\right), 37.77(\mathrm{CH}), 48.97\left(\mathrm{CH}_{2}\right), 95.37(\mathrm{C}), 112.75(\mathrm{CH}), 118.66(\mathrm{C}), 120.04$ $(\mathrm{CH}), 120.59(\mathrm{CH}), 125.05(\mathrm{C}), 125.27(\mathrm{CH}), 125.47(2 \times \mathrm{CH}), 125.59(\mathrm{C}), 127.00(\mathrm{C}), 129.66$ $(\mathrm{CH}), 134.54(\mathrm{CH}), 136.86(\mathrm{C}), 150.69(\mathrm{C}), 159.67(\mathrm{C}=\mathrm{N})$ and $170.37(\mathrm{C}=\mathrm{O})$ ppm; Found: $\mathrm{C}$, 77.27, $\mathrm{H}, 6.12, \mathrm{~N}, 7.99 \% \mathrm{C}_{22} \mathrm{H}_{20} \mathrm{~N}_{2} \mathrm{O}_{2}$ requires $\mathrm{C}, 76.71, \mathrm{H}, 5.81, \mathrm{~N}, 8.14 \%$; Found: $\mathrm{M}$ 344.1524 (1.4\%) $\mathrm{C}_{22} \mathrm{H}_{20} \mathrm{~N}_{2} \mathrm{O}_{2}$ requires 344.1525. 


\section{Acknowledgements}

We thank the University of Jordan and The British Council (M.O.T.) for financial support.

\section{References}

1. Active Oxygen in Chemistry, Foote, C.S.; Valentine, J.S.; Greenberg, A.; Liebman, J.F., Eds; Chapman and Hall: New York, 1995.

2. (a) Criegee, R. In Houben-Weyl Methoden der organischen Chemie, $4^{\text {th }}$ Edn, Oxygen Compounds III, Thieme: Stuttgart, 1952; Vol. 8. (b) Karnojitzky, V. Russ. Chem. Rev. (Engl. Transl.) 1981, 50, 888.

3. (a) Doering, W. von E.; Haines, R.M. J. Am. Chem. Soc. 1954, 76, 482. (b) Elkik, E. Bull Soc. chim. France 1959, 933. (c) Baldwin, J.E.; Barton, D.H.R.; Faulkner, D.J.; Templeton, J.F. J. Chem. Soc.1962, 4743.

4. Crombie, L.; Godin, P.J. J. Chem. Soc. 1961, 2861.

5. (a) Bailey, E.J.; Elks, J.; Barton, D.H.R. Proc. Chem. Soc. 1960, 214. (b) Bailey, E.J.; Barton, D.H.R.; Elks, J.; Templeton, J.F. J. Chem. Soc. 1962, 1578.

6. Barton, D.H.R.; Werstiuk, N.H. J. Chem. Soc. (C) 1968, 1148.

7. Barton, D.H.R.; Pradhan, S.K.; Sternhell, S.; Templeton, J.F. J. Chem. Soc. 1961, 255.

8. (a) Cardwell, H.M.E.; McQuillin, F.J. J. Chem. Soc. 1955, 525. (b) Howe, R.; McQuillin, F.J. J. Chem. Soc. 1958, 1513.

9. Paquette, L.A.; DeRussy, D.T.; Pegg, N.A.; Taylor, R.T.; Zydowsky, T.M. J. Org. Chem. 1989, 54, 4576. See also the extensive list of references for additional examples of the oxidation of ketones using dioxygen.

10. Wender, P.A.; Glass, T.E.; Krauss, N.E.; Muhlebach, M.; Peschke, B.; Rawlins, D.B. J. Org. Chem. 1996, 61, 7662.

11. (a) Avramoff, M.; Sprinzak, Y. Proc. Chem. Soc. 1962, 150. (b) Gersmann, H.R.; Nieuwenhuis, H.J.W.; Bickel, A.F. Proc. Chem. Soc. 1962, 279. (c) Gersmann, H.R.; Nieuwenhuis, H.J.W.; Bickel, A.F. Tetrahedron Lett.1963, 1383.

12. Baldwin, J.E.; Barton, D.H.R.; Sutherland, J.K. J. Chem. Soc. 1964, 3312.

13. (a) Nishio, T. J. Chem. Soc., Perkin Trans. 1 1991, 1717. (b) Yu, Q.-s.; Li, Y.-q.; Luo, W.m.; Brossi, A. Heterocycles 1993, 36, 1791.

14. Adam, W.; Metz, M.; Prechtl, F.; Renz, M. Synthesis 1994, 563.

15. Semple, J.E.; Rydzewski, R.M.; Gardner, G. J. Org. Chem. 1996, 61, 7967.

16. Russell, G.A.; Moye, A.J.; Nagpal, K. J. Am. Chem. Soc. 1962, 84, 4154.

17. Horning, D.E.; Lacasse, G.; Muchowski, J.M. Can. J. Chem 1971, 49, 246; See also Larock, R.C. Comprehensive Organic Transformations VCH: New York, 1989; p 488.

18. Heaney, H.; Taha, M.O.; Slawin, A.M.Z. Tetrahedron Lett. 1997, 38, 3051.

19. Ke-Qing, L.; Gang, J.; Hu, C.; Jian-Hua, X. Tetrahedron Lett. 1998, 37, 2381 
20. Heaney, H.; Taha, M.O. Synlett 1996, 820.

21. (a) Peterson, S.; Heitzer, H. Liebigs Ann. Chem. 1978, 283. (b) Wrobel, J.; Dietrich, A. Heterocycles, 1994, 38, 1823.

22. Druliner, J.D.; Hobbs, F.W.; Seidel, W.C. J. Org. Chem. 1988, 53, 700.

23. (a) Gardner, J.N.; Carlon, F.E.; Gnoj, O. J. Org. Chem., 1968, 33, 32947. (b) Gardner, J.N.; Poppen, J.L.; Carlon, F.E.; Gnoj, O.; Herzog, H. J. Org. Chem. 1968, 33, 3695.

24. Shamma, M. The Isoquinoline Alkaloids Academic Press: New York, 1972; Ch. 19.

25. Flack, J.R.; Manna, S. Tetrahedron Lett. 1981, 22, 619.

26. (a) Prager, R.H.; Tippett, J.M.; Ward, A.D. Austal. J. Chem. 1981, 34, 1085. (b) Clarke, S.I.; Kasum, B.; Prager, R.H.; Ward, A.D. Austral. J. Chem. 1983, 36, 2493.

27. Keay, B.A.; Dibble, P.W. In Comprehensive Heterocyclic Chemistry II, Katritzky, A.R.; Rees, C.W.; Scriven, E.F.V., Eds; Pergamon: Oxford, 1996, Vol 2, Bird, C.W., Ed; Ch. 2.08; pp 395.

28. Muchowski, J.M. Can. J. Chem 1969, 47, 857.

29. Huffman, J.W.; Miller, E.G. J. Org. Chem 1960, 25, 90.

30. Solomina, L.P.; Grigoryan, R.T.; Markaryan, E.A. Arm. Khim. Zh 1978, 31, 345; Chem. Abs 1978, 89, 197265g.

31. Pulvermacher, G. Chem. Ber 1887, 20, 2492. 